\title{
Delaunay graphs of point sets in the plane with respect to axis-parallel rectangles
}

\author{
Xiaomin Chen, János Pach ${ }^{\dagger}$ Mario Szegedy $\ddagger$ and Gábor Tardos ${ }^{\S}$
}

\begin{abstract}
To solve frequency assignment problems in cellular telephone networks, Even, Lotker, Ron, and Smorodinsky (FOCS 02) introduced the notion of conflict-free colorings in various geometrically defined hypergraphs. They initiated the investigation of the special case when the vertex set of the hypergraph is a set $P$ of $n$ points in the plane, and the hyperedges are those subsets of $P$ that can be obtained by intersecting $P$ with an axis-parallel rectangle. The 2-element subsets of $P$ satisfying this condition form (the edge set of) the Delaunay graph $D(P)$ associated with $P$. The problem of estimating the minimum number of colors in a conflict-free coloring leads to the following question: Does there exist a constant $c>0$ such that the Delaunay graph of any set of $n$ points in the plane contains an independent set of size at least $c n$ ? We answer this question in the negative. We also show that for a set $P$ of $n$ randomly and uniformly selected points in the unit square, $D(P)$ has an independent set of size at least $\mathrm{cn} / \log n$, with probability tending to 1 . We generalize these results to solve a problem in geometric discrepancy theory.
\end{abstract}

\section{Delaunay graphs and conflict-free colorings}

The Delaunay graph associated with a set of points $P$ in the plane is a graph $D(P)$ whose vertex set is $P$ and whose edge set consists of those pairs $\{p, q\} \subset P$ for which there exists a closed disk that contains $p$ and $q$, but does not contain any other element of $P$. The Delaunay graph of $P$ is a planar graph and its dual is

\footnotetext{
${ }^{*}$ Google, 76 Ninth Avenue, New York, NY, 10011.

${ }^{\dagger}$ City College, CUNY and Courant Institute, NYU, 251 Mercer Street, New York, NY 10012. Supported by NSF grant CCF-05-14079 and grants from NSA, PSC-CUNY, Hungarian Research Foundation, and BSF.

${ }^{\ddagger}$ Rutgers University, 110 Frelinghuysen Road Piscataway, NJ 08854-8019. Supported by NSF grant 0105692 .

${ }^{\S}$ Simon Fraser University, 8888 University Drive, Burnaby, B.C., Canada V5A 1S6 and Rényi Institute, H-1055 Budapest, Reáltanoda utca 13-15, Hungary. Supported by NSERC grant 329527.
} 
the Dirichlet-Voronoi diagram of $P$ (see, e.g., [BKOS00]). As any other planar graph, $D(P)$ contains an independent set of size at least $|P| / 4$. It was discovered by Even, Lotker, Ron, and Smorodinsky [ELRS03] that this fact easily implies that any set $P$ of $n$ points in the plane has a conflict-free coloring with respect to discs, which uses at most $O(\log n)$ colors, that is, a coloring with the property that any closed disk $C$ with $C \cap P \neq \emptyset$ has an element whose color is not assigned to any other element of $C \cap P$. Here, the logarithmic bound is tight for every point set [PaT03].

The question was motivated by a frequency assignment problem in cellular telephone networks. The points correspond to base stations interconnected by a fixed backbone network. Each client continuously scans frequencies in search of a base station within its (circular) range with good reception. Once such a base station is found, the client establishes a radio link with it, using a frequency not shared by any other station within its range. Therefore, a conflict-free coloring of the points corresponds to an assignment of frequencies to the base stations, which enables every client to connect to a base station without interfering with the others. For many results on conflict-free colorings, consult [AlS06], [FiLM05], [HaS05].

The same scheme can be used to construct conflict-free colorings of point sets with respect to various other families of geometric figures. In general, let $P$ be a set of points in $\mathbf{R}^{d}$, and let $\mathcal{C}$ be a family of $d$-dimensional convex bodies. Define the Delaunay graph $D_{\mathcal{C}}(P)$ of $P$ with respect to $\mathcal{C}$ on the vertex set $P$ by connecting two elements $p, q \in P$ with an edge if and only if there is a member of $\mathcal{C}$ that contains $p$ and $q$, but no other element of $P$. The existence of large independent sets in such graphs implies that $P$ has a conflict-free coloring with respect to $\mathcal{C}$, which uses a small number of colors. That is, a coloring with the property that any member $C \in \mathcal{C}$ with $C \cap P \neq \emptyset$ has an element whose color is not assigned to any other element of $C \cap P$.

In this note, we consider this problem in the special case when $\mathcal{C}$ is the family of axis-parallel boxes. The maximum size of an independent set of vertices in a graph $G$ is called the independence number of $G$, and is usually denoted by $\alpha(G)$ in the literature. Smorodinsky et al. [ELRS03], [HaS05] asked whether the Delaunay graph of every set of $n$ points in the plane with respect to axis-parallel rectangles has independence number at least $c n$, for an absolute constant $c>0$. In Section 3, we give a negative answer to this question. More precisely, we establish

Theorem 1. There are n-element point sets in the plane such that the independence numbers of their Delaunay graphs with respect to axis-parallel rectangles are at most $O\left(n \frac{\log ^{2} \log n}{\log n}\right)$.

In fact, a randomly and uniformly selected set of $n$ points in the unit square will meet the requirements with probability tending to 1 . 
For randomly selected point sets, this result is not far from being best possible. In Section 2, we prove

Theorem 2. The expected value of the independence number of a randomly and uniformly selected $n$-element point set in the units square is $\Omega\left(\frac{n}{\log n}\right)$.

For general point sets, we know only a very weak bound: the independence number of the Delaunay graph of any set of $n$ points in the plane with respect to axis-parallel rectangles is at least $\Omega(\sqrt{n \log n})$. This only implies that any set of $n$ points in the plane admits a conflict-free coloring using $O(\sqrt{n / \log n})$ colors, with respect to the family of all axis-parallel rectangles.

In discrepancy theory [BeCh87], [Ch00], [Ma99], there are plenty of results that indicate some unavoidable irregularities in geometric configurations. In Section 3, we generalize Theorem 1 . Our results immediately imply

Theorem 3. For any constants $c, d>1$, a randomly and uniformly selected set $P$ of $n$ points in the unit square almost surely has the following property. For any coloring of the elements of $P$ with c colors, there always exists an axis-parallel rectangle with at least $d$ points in its interior, all of which have the same color.

\section{Delaunay graphs of random point sets}

The aim of this section is to prove Theorem 2 .

Let $P=\left\{\left(x_{i}, y_{i}\right): 1 \leq i \leq n\right\}$ be a point set in the unit square, whose no two elements share the same $x$-coordinate or $y$-coordinate. Clearly, the Delaunay graph $D(P)$ with respect to axis-parallel rectangles depends only on the relative position of the points in $P$ and not on their actual coordinates. That is, there exists a permutation $\pi:\{1,2, \ldots, n\} \rightarrow\{1,2, \ldots, n\}$ such that for the set $P^{\prime}=\{(i, \pi(i)): 1 \leq i \leq n\}$ we have $D(P)=D\left(P^{\prime}\right)$. Moreover, for a random set of points in the square, the corresponding permutation $\pi$ is uniformly random. With a slight abuse of notation, we write $D(\pi)$ for the Delaunay graph $D(P)=D\left(P^{\prime}\right)$. In our arguments about Delaunay graphs of randomly selected point sets in the square, it will be convenient to consider the graph $D(\pi)$ for a random permutation $\pi$.

Lemma 1. Let $\pi:\{1,2, \ldots, n\} \rightarrow\{1,2, \ldots, n\}$ be a random permutation, and let $\overline{d e g}(D(\pi))$ denote the average degree of the vertices of the Delaunay graph $D(\pi)$. The expected value of the average degree satisfies

$$
\mathrm{E}(\overline{\operatorname{deg}}(D(\pi)))=\Theta(\log n) .
$$


Proof. Two points $p_{i}=(i, \pi(i))$ and $p_{j}=(j, \pi(j))$ with $i<j$ are connected by an edge in $D(P)$ if and only if $\pi(i)$ and $\pi(j)$ are consecutive elements in the natural ordering of the set $S=\{\pi(k) \mid i \leq k \leq j\}$. Among all $\left(\begin{array}{c}j-i+1 \\ 2\end{array}\right)$ pairs of elements in this set, precisely $j-i$ consist of consecutive elements. Clearly, after fixing $\pi(k)$ for $k<i$ or $k>j$, the pair $\{\pi(i), \pi(j)\}$ is equally likely to be any one of the pairs in $S$. Therefore, the probability that $p_{i}$ and $p_{j}$ are connected is equal to

$$
\frac{j-i}{\left(\begin{array}{c}
j-i+1 \\
2
\end{array}\right)}=\frac{2}{j-i+1} .
$$

Thus, the expected number of edges in $D(P)$ is

$$
\sum_{l=1}^{n-1} \frac{2(n-l)}{l+1}=(2 n+2) \sum_{l=1}^{n} \frac{1}{l}-4 n=\Theta(n \log n) .
$$

Obviously, Theorem 2 is equivalent to

Theorem 2'. Let $\pi$ be a random permutation of $\{1,2, \ldots, n\}$. The expected value of the independence number of the Delaunay graph $D(\pi)$ with respect of axisparallel rectangles satisfies

$$
\mathrm{E}[\alpha(D(\pi))]=\Omega\left(\frac{n}{\log n}\right) .
$$

Proof. According to Turán's theorem, any graph with $n$ vertices and average degree $d$ has an independent set of size at least $\frac{n}{d+1}$. Thus, we have

$$
\alpha(D(\pi)) \geq \frac{n}{\overline{\operatorname{deg}}(D(\pi))+1} .
$$

By the convexity of the $x \rightarrow n /(x+1)$ function for $x \geq 0$, we have

$$
\mathrm{E}[\alpha(D(\pi))] \geq \frac{n}{\mathrm{E}[\overline{\operatorname{deg}}(D(\pi))+1}
$$

and the theorem follows by Lemma 1. 


\section{Proof of Theorem 1}

We reformulate and prove Theorem 1 in a more precise form.

Theorem 1'. Let $P$ be a set of $n$ randomly and uniformly selected points in the square $[0,1]^{2}$. Then there exists a constant $c$ such that

$$
\operatorname{Prob}_{n \rightarrow \infty}\left(\alpha(D(P))<c \frac{n \log ^{2} \log n}{\log n}\right) \rightarrow 1 .
$$

Proof. The points $p_{i} \in P$ will be defined in two steps. First we select the $x$ coordinates from the interval $[0,1]$ uniformly at random. With probability 1 , all the $x$ coordinates are distinct. Let us relabel the points so that

$$
0 \leq x_{1}<x_{2}<\cdots<x_{n} \leq 1
$$

In the second step, we select the $y$-coordinates of $p_{i}=\left(x_{i}, y_{i}\right)$ uniformly and independently from $[0,1]$. Note that, after the $x_{i}$ 's have been fixed, the edge set of the Delaunay graph $D(P)$ depends only on the relative order of the $y_{i}$ 's.

The coordinates $y_{i}$ are generated as follows. Fix an integer $L \geq 2$ to be specified later. We write the numbers $y_{i} \in[0,1]$ in base $L$ :

$$
y_{i}=\left(0 . d_{i}^{(1)} d_{i}^{(2)} \ldots\right)_{L} .
$$

The digits $d_{i}^{(t)}$ of $y_{i}$ are chosen independently and uniformly from the set $\{0, \ldots, L-1\}$. For $t \geq 1$, denote by $y_{i}^{(t)}$ the truncated $L$-ary fraction of $y_{i}$, consisting of $t-1$ digits after 0 :

$$
y_{i}^{(t)}=\left(0 . d_{i}^{(1)} \ldots d_{i}^{(t-1)}\right)_{L} .
$$

The digits of $y_{i}$ will be chosen one by one. At stage $t$, we determine $d_{i}^{(t)}$ (and, hence, $\left.y_{i}^{(t+1)}\right)$, for all $i$. Note that before stage $t$, the truncated fractions $y_{i}^{(t)}$ have already been fixed. As soon as we complete stage $t$, we know the $y$-coordinates of the points $p_{i}$ up to an error of at most $L^{-t}$. If $y_{i}^{(t+1)}=y_{j}^{(t+1)}$, then the relative order of $y_{i}$ and $y_{j}$ has not yet been decided. Otherwise, if we have $y_{i}^{(t+1)}<y_{j}^{(t+1)}$, say, then $y_{i}<y_{j}$ holds in the final configuration.

Let $1 \leq i<j \leq n$ be fixed. Suppose that for some $t$, the following two conditions are satisfied:

$$
\text { 1. } y_{i}^{(t+1)}=y_{j}^{(t+1)} \text {, }
$$


2. $y_{k}^{(t+1)} \neq y_{i}^{(t+1)}$ holds for all $k$ satisfying $i<k<j$.

Then the rectangle $\left[x_{i}, x_{j}\right] \times\left[y_{i}^{(t+1)}, y_{i}^{(t+1)}+L^{-t}\right)$ contains $p_{i}$ and $p_{j}$, but no other element of $P$. Thus, in this case, $p_{i}$ and $p_{j}$ are connected in $D(P)$, and we say that this edge is forced at stage $t$. Although $D(P)$ may contain many edges that are not forced at any stage, we are going to use only forced edges in proving our upper bound on the independence number of $D(P)$.

Let us fix a subset $I \subset\{1, \ldots, n\}$, and let $Q=Q(I)=\left\{p_{i}: i \in I\right\}$. We want to estimate from above the probability that $Q$ is an independent set in $D(P)$.

Let $t \geq 1$, and consider stage $t$ of our selection process. Before this stage, $y_{i}^{(t)}$ has been fixed for every $i$. For any $L$-ary fraction $y$ of the form $y=\left(0 . d^{(1)} d^{(2)} \cdots d^{(t-1)}\right)_{L}$, define a subset $H_{y} \subseteq\{1, \ldots, n\}$ by

$$
H_{y}=\left\{1 \leq i \leq n: y_{i}^{(t)}=y\right\}
$$

Obviously, these sets partition $\{1, \ldots, n\}$, and hence $I$, into at most $L^{t-1}$ nonempty parts. If two indices $i, j \in I$ are consecutive elements of the same part $H_{y} \cap I$, then we call them neighbors. That is, $i<j$ are neighbors if

1. $y_{i}^{(t)}=y_{j}^{(t)}=y$ holds for some $y$, and

2. $H_{y} \cap\{k \in I: i<k<j\}=\emptyset$.

For any two neighbors $i, j \in H_{y}(i<j)$, define

$$
S_{i, j}=\left\{k \in H_{y}: i<k<j\right\} .
$$

Two neighbors $i, j \in I(i<j)$ are called close neighbors if $\left|S_{i, j}\right| \leq L$.

If there are two close neighbors $i, j \in I$ such that the $\left\{p_{i}, p_{j}\right\}$ is an edge of $D(P)$ forced at stage $t$, then $Q$ is not an independent set in $D(P)$ and we say that $Q$ fails at stage $t$. Otherwise, $Q$ is said to survive stage $t$, and we indicate this fact by writing $Q \curvearrowright t$.

Let $i<j$ be a pair of close neighbors. Note that $\left\{p_{i}, p_{j}\right\}$ is an edge of $D(P)$ forced in stage $t$ if and only if $d_{i}^{(t)}=d_{j}^{(t)}$, but $d_{i}^{(t)} \neq d_{k}^{(t)}$ holds for all $k \in S_{i, j}$. The probability of this event is

$$
\operatorname{Prob}\left(\left\{p_{i}, p_{j}\right\} \text { is forced at stage } t\right)=\frac{1}{L}\left(1-\frac{1}{L}\right)^{\left|S_{i, j}\right|} .
$$

Taking into account that $\left|S_{i, j}\right| \leq L$, we obtain

$$
\operatorname{Prob}\left(\left\{p_{i}, p_{j}\right\} \text { is forced at stage } t\right) \geq \frac{1}{4 L} \text {. }
$$


Notice that, assuming a fix outcome of previous stages (i.e., $p_{k}^{(t)}$ is fixed for all $k)$, the presence of edges $\left\{p_{i}, p_{j}\right\}$ forced at stage $t$ are independent for all neighbors. Thus,

$$
\operatorname{Prob}\left(Q \curvearrowright t \mid \text { outcome of stages } t^{\prime}<t\right) \leq\left(1-\frac{1}{4 L}\right)^{m} \leq e^{-\frac{m}{4 L}},
$$

where $m$ stands for the number of pairs $i, j \in I$ that are close neighbors before stage $t$.

Obviously, every $i \in I$, except the last element in each set $H_{y}$, has exactly one neighbor $j>i$. As the sets $S_{i, j}$ are pairwise disjoint for different pairs of neighbors $i<j$, there are fewer than $\frac{n}{L}$ pairs that are neighbors but not close neighbors. Thus, we have

$$
m>|I|-\frac{n}{L}-L^{t-1} .
$$

If $t \leq \log n / \log L$ and $|I| \geq 3 n / L$, we have $m \geq n / L$, and thus

$$
\operatorname{Prob}\left(Q \curvearrowright t \mid \text { outcome of stages } t^{\prime}<t\right) \leq e^{-\frac{n}{4 L^{2}}} .
$$

As the above bound applies assuming any set of choices made at previous stages, so in particular, it applies to the conditional probability that $Q$ survives stage $t$, given that it has survived all previous stages:

$$
\operatorname{Prob}\left(Q \curvearrowright t \mid Q \curvearrowright t^{\prime} \text { for all } t^{\prime}<t\right) \leq\left(1-\frac{1}{4 L}\right)^{m} \leq e^{-\frac{n}{4 L^{2}}}
$$

Taking the product of these estimates for all $t \leq \log n / \log L$, we obtain

$\operatorname{Prob}(Q$ survives the first $\lfloor\log n / \log L\rfloor$ stages $) \leq \exp \left(-\frac{n}{4 L^{2}}\left(\frac{\log n}{\log L}-1\right)\right)$.

The last bound is valid for any set $Q=Q(I) \subseteq P$, where $I \subset\{1, \ldots, n\}$ satisfies $|I| \geq 3 n / L$. Letting

$$
L=\left\lfloor\frac{\log n}{100 \log ^{2} \log n}\right\rfloor \quad \text { and } \quad a=\left\lceil\frac{3 n}{L}\right\rceil,
$$

we can conclude that

$$
\begin{aligned}
\operatorname{Prob}(\alpha(D(P)) \geq a) & \leq \sum_{Q \subset P,|Q|=a} \operatorname{Prob}(Q \text { survives all stages }) \\
& \leq\left(\begin{array}{c}
n \\
a
\end{array}\right) \exp \left(-\frac{n}{4 L^{2}}\left(\frac{\log n}{\log L}-1\right)\right) \\
& \rightarrow 0
\end{aligned}
$$

as required. 


\section{Discrepancy in colored random point sets}

In this section, we strengthen Theorem 1.

Definition 1. Given an integer $d>1$ and a finite point set $P$ in the plane, a subset $Q \subseteq P$ is called $d$-independent if there is no axis-parallel rectangle $R$ such that $|R \cap P|=d$ and $R \cap P \subseteq Q$. Let $\alpha_{d}(P)$ denote the size of the largest $d$-independent subset of $P$.

According to this definition, a subset of $P$ is 2 -independent if and only if it is an independent set in the Delaunay graph $D(P)$ associated with $P$. In particular, we have $\alpha_{2}(P)=\alpha(D(P))$.

Obviously, if a set is $d$-independent for some $d>1$, then it is also $d^{\prime}$ independent for any $d^{\prime}>d$. Therefore, $\alpha_{d}(P)$ is increasing in $d$.

Theorem 3 is a direct corollary to

Theorem 4. A randomly and uniformly selected set $P$ of $n$ points in the unit square almost surely satisfies

$$
\alpha_{d}(P)=O\left(\frac{d n \log ^{2} \log n}{\log ^{1 /(d-1)} n}\right) .
$$

Proof. We modify the proof of Theorem 1. Pick the random points $p_{i}=\left(x_{i}, y_{i}\right) \in$ $P$ according to the same multi-stage model as in the previous section, and define the truncated fractions $y_{i}^{(t)}$ that approximate $y_{i}$ in exactly the same way as before.

Fix a subset $I \subseteq\{1, \ldots, n\}$, and let $Q=Q(I)=\left\{p_{i}: i \in I\right\}$. Just like in the proof of Theorem 1 , analyze a fixed stage $t$ of the selection process, by introducing the sets $H_{i}$.

Instead of using the notion of neighbors, we need a new definition. For any two elements $i, j \in I(i<j)$ such that $y_{i}^{(t)}=y_{j}^{(t)}=y$ for some $y$, introduce the sets

$$
T_{i, j}=\left\{k \in H_{y} \cap I: i \leq k \leq j\right\} \quad \text { and } \quad S_{i, j}=\left\{k \in H_{y} \backslash I: i<k<j\right\} .
$$

The numbers $i$ and $j$ are called $d$-neighbors if $\left|T_{i, j}\right|=d$. The pair $\{i, j\}$ of $d$ neighbors is called a pair of close d-neighbors if $\left|S_{i, j}\right| \leq L$.

We say that the pair of close $d$-neighbors $\left\{p_{i}, p_{j}\right\}$ fails at stage $t$ if at this stage the $y$-coordinates of all points $p_{k}$ with $k \in T_{i, j}$ receive the same new digit $d_{k}^{(t)}=\delta$, but the $y$-coordinate of no point $p_{\ell}$ with $\ell \in S_{i, j}$ receives this digit. The probability of this event is exactly

$$
L^{1-d}\left(1-\frac{1}{L}\right)^{\left|S_{J}\right|} \geq L^{1-d}\left(1-\frac{1}{L}\right)^{L} \geq \frac{1}{4 L^{d-1}}
$$


Obviously, if any pair $\left\{p_{i}, p_{j}\right\}$ fails at stage $t$, then $Q$ cannot be $d$-independent. In this case, we say that $Q$ fails at stage t. Otherwise, $Q$ is said to have survived stage $t$, and we write $Q \curvearrowright t$.

The failures of certain pairs at a given stage are not independent events. However, they are independent for any collection of close $d$-neighbor pairs $(i, j)$ with the property that the corresponding sets $T_{i, j}$ are pairwise disjoint. To find such a

collection consisting of many pairs, select at least $\frac{\left|H_{y} \cap I\right|}{d-1}-1$ pairs of $d$-neighbors from each $H_{y}$ with pairwise disjoint sets $T_{i, j}$, and thus a total of at least $\frac{|I|}{d-1}-L^{t-1}$ pairs. Since the corresponding sets $S_{i, j}$ are pairwise disjoint, all but at most $n / L$ of them are close $d$-neighbors. Thus, as long as $|I| \geq 3(d-1) n / L$ and $t \leq \log n / \log L$, we obtain collection of

$$
m \geq \frac{|I|}{d-1}-L^{t-1}-\frac{n}{L} \geq \frac{n}{L}
$$

close $d$-neighbors with the required property.

If any pair of this collection fails at stage $t$, then $Q$ fails at this stage. As in the proof of Theorem 1, we have

$$
\operatorname{Prob}\left(Q \curvearrowright t \mid Q \curvearrowright t^{\prime} \text { for all } t^{\prime}<t\right) \leq e^{-\frac{n}{4 L^{d}}}
$$

and

$$
\operatorname{Prob}(Q \text { survives all stages }) \leq \exp \left(-\frac{n}{4 L^{d}}\left(\frac{\log n}{\log L}-1\right)\right) .
$$

Letting

$$
L=\left\lfloor\frac{\log ^{1 /(d-1)} n}{100 \log ^{2} \log n}\right\rfloor \quad \text { and } \quad a=\left\lceil\frac{3(d-1) n}{L}\right\rceil \text {, }
$$

we obtain

$$
\operatorname{Prob}(\alpha(D(P)) \geq a)<\left(\begin{array}{l}
n \\
a
\end{array}\right) \exp \left(-\frac{n}{4 L^{d}}\left(\frac{\log n}{\log L}-1\right)\right) \rightarrow 0 .
$$

\section{Concluding remarks, open problems}

The notion of Delaunay graphs for axis-parallel boxes naturally generalizes to higher dimensions. An easy extension of the proof of Theorem 2 proves that for any fixed $d$, the Delaunay graph of randomly and uniformly selected points in the 
$d$-dimensional unit cube has expected average degree $O\left((\log n)^{d}\right)$. This implies that random Delaunay graphs have independent sets of size $n^{1-o(1)}$ in higher dimensions, too. All lower bounds that apply to dimension $d$ also apply to every larger dimension. This can easily be seen by projecting a $d$-dimensional point sets to a coordinate hyperplane. Delaunay graphs can only lose edges under this operation.

In general, by repeated application of the Erdős-Szekeres lemma it is easy to show that the independence number of the Delaunay graph of any set of $n$ points in $d$-dimensions, with respect to axis-parallel boxes, is at least $\Omega\left(n^{1 / 2^{d-1}}\right)$. As far as we know, no significant improvement on this bound is known, although the truth may well be $\Omega_{d}(n-o(1))$, for any fixed $d$.

Returning to the plane, it is not hard show that the expected number of $d$ tuples $T$ in a randomly and uniformly selected set $P$ of $n$ points in the plane, for which there exists an axis-parallel rectangle whose intersection with $P$ is $T$, is $\Theta\left(d^{2} n \log n\right)$. By a result of Spencer [Sp72], any $d$-uniform hypergraph with $n$ vertices and $\Theta(n k)$ edges has an independent set of size $\Omega\left(n / k^{1 /(d-1)}\right)$. Therefore, $P$ contains a $d$-independent subset of size $\Omega\left(n / \log ^{1 /(d-1)} n\right)$. This is within $O\left(\log ^{2} \log n\right)$ of our upper bound.

\section{References}

[AlS06] N. Alon and S. Smorodinsky: Conflict-free colorings of shallow discs, in: Proc. 22nd Ann. ACM Symp. on Computational Geom (SoCG 2006), to appear.

[BeCh87] J. Beck, and W. Chen: Irregularities of Distribution. Cambridge Tracts in Mathematics 89, Cambridge University Press, Cambridge, 1987.

[BKOS00] M. de Berg, M. van Kreveld, M. Overmars, and O. Schwarzkopf: Computational Geometry. Algorithms and Applications. 2nd ed., Springer-Verlag, Berlin, 2000.

[Ch00] B. Chazelle: The Discrepancy Method. Randomness and Complexity, Cambridge University Press, Cambridge, 2000.

[ClS89] K. L. Clarkson and P. W. Shor: Applications of random sampling in computational geometry. II, Discrete Comput. Geom. 4 (1989), 387-421.

[ELRS03] G. Even, Z. Lotker, D. Ron, and S. Smorodinsky: Conflict-free colorings of simple geometric regions with applications to frequency assignment in cellular networks, SIAM J. Comput. 33 (2003), 94-136. 
[FiLM05] A. Fiat, M. Levy, J. Matoušek, E. Mossel, J. Pach, M. Sharir, S. Smorodinsky, U. Wagner, and E. Welzl: Online conflict-free coloring for intervals, in: Proc. 16th Ann. ACM-SIAM Symp. on Discrete Algorithms (SODA 2005), 545-554.

[HaS05] S. Har-Peled and S. Smorodinsky: Conflict-free coloring of points and simple regions in the plane, Discrete \& Computational Geometry 34 (2005), 47-70.

[KeL86] K. Kedem, R. Livne, J. Pach, and M. Sharir: On the union of Jordan regions and collision-free translational motion amidst polygonal obstacles, Discrete Comput. Geom. 1 (1986), 59-71.

[Ma99] J. Matoušek: Geometric Discrepancy. An Illustrated Guide. Algorithms and Combinatorics 18, Springer-Verlag, Berlin, 1999.

[PaT03] J. Pach and G. Tóth: Conflict-free colorings, in: Discrete and Computational Geometry, Algorithms Combin., Vol. 25, Springer, Berlin, 2003, 665-671.

[Sm06] S. Smorodinsky: On the chromatic number of some geometric hypergraphs, in: Proc. 16th Ann. ACM-SIAM Symp. on Discrete Algorithms (SODA 2006), 316-323.

[Sp72] J. Spencer: Turán's theorem for k-graphs, Discrete Math. 2 (1972), 183186. 